\title{
Analysis of the Business Models of the Intelligent and Connected Vehicle Industry
}

\author{
Fuquan Zhao ${ }^{1,2}$, Hong Tan ${ }^{1,2}$, Zongwei Liu ${ }^{1,2, a}$ \\ ${ }^{1}$ State Key Laboratory of Automotive Safety and Energy, Tsinghua University, Beijing 100084, China \\ 2 Tsinghua Automotive Strategy Research Institute, Tsinghua University, Beijing 100084, China
}

\begin{abstract}
Intelligent connected vehicles (ICV) are recognized as a great opportunity with huge social benefits by the global auto industry. Governments of various countries attach great importance to them, and traditional Original Equipment Manufacturer (OEM) and technology companies are also introducing them into consumers' lives by virtue of various business models, thereby generating practical value. The business model plays a decisive role in determining whether a company can share the ICV market cake and its future position in the industry. More importantly, it will also determine how fast the ICVs can become reality from imagination. In this paper, the ICV industry ecosystem was sorted out, the business models of ICVs adopted by mainstream OEMs were analyzed, and five typical business models were summarized, i.e. platform model, self-transformation model, traditional Tier1 dependence model, alliance model, and outsourcing model. On this basis, the SWOT analysis method was adopted to systematically analyze the internal and external advantages and disadvantages of the five business models, and forward insights on enterprises' developing and maintaining the competitive advantages in the ICV industry were proposed from five perspectives of technology, market timing, customer experience, brand, and data.
\end{abstract}

\section{Introduction}

At present, the industrial revolution triggered by the new round of global scientific and technological revolution has brought unprecedented opportunities and challenges to the development of the automotive industry [1]. The all-round changes in the automotive industry have given birth to historic opportunities [2]. ICVs have huge social benefits. The deployment of ICVs is regarded as a significant solution to improve road safety, transportation management, and energy efficiency, of which the total benefits may reach 13.25 to 24.02 trillion CNY in 2050 in China $[3,4,5]$.

With the vigorous promotion by the governments in the United States, the United Kingdom, Germany, Japan, China and other countries, ICVs have also gone through a journey from "may be realized" to "will be realized" to "will definitely come true". Traditional OEMs and technology companies are racing to make ICVs a reality through multiple business models. Based on SAE's classification of smart vehicles [6], in industrial practice, some revolutionary companies advocate skipping L3 and heading directly to L4 and aim at emerging markets with great potential such as mobility services and automatic distribution services. However, some conservative companies advocate step-by-step evolution from L3 and believe that L3 products have commercial value and can solve the users' pain points in certain scenarios [7].
The auto industry is characterized by a large scale, a strong driving effect, a wide coverage of fields and influence, and highly intensive capital, technology and talent [8]. Not every player can reach its destination because the business model it chooses will not only determine whether and when it can reach the destination, but also the fate after it reaches the destination. Automobile manufacturers need to create a new market for these vehicles substantiating real value to customers [9]. The business model has a huge impact on OEMs. It is an important source of competitiveness for smart car companies [10], and an important driving factor for the breakthrough development of OEMs in the ICV sector. It is identified that business model is important in technology intensive industries such as autonomous vehicle [11].

The structure of this paper is shown as follows: section 2 sorts out the ICV industry ecosystem; section 3 summarizes and analyses the five business models for the ICV industry; section 4 summarizes the current competitive landscape of the ICV industry; and section 5 gives suggestions on how companies can develop and maintain a competitive advantage.

\section{ICV industry ecosystem}

The ICV concept represents the commanding heights of prospective automotive product shape and technology,

\footnotetext{
${ }^{\mathrm{a}}$ Corresponding author: tasri01@mail.tsinghua.edu.cn
} 
which will pose significant impacts on the value chain of future automotive industry, especially in China [12]. ICV, a large and holistic concept, consists of a sustainable development ecosystem formed by various functional parts, including ICV development and manufacturing groups, market introduction groups, and legal, insurance, and infrastructure support. No company can succeed on its own, and cooperation is imperative. Looking more closely at the composition of the entire ICV industry ecosystem, there are cybersecurity solution providers represented by Harman, big data service providers represented by IBM, V2X suppliers represented by Huawei, map suppliers represented by Here, computing platform providers represented by Nvidia, chip suppliers represented by NXP, sensor manufacturers represented by Valeo, ICV software startups represented by nuTonomy, ICV platform suppliers represented by Baidu, traditional T1 suppliers represented by Bosch, new OEMs represented by Tesla, and traditional OEMs represented by GM, who together constitute the ICV development and manufacturing group. In addition, ride sharing companies represented by Uber and Lyft, and ICV maintenance companies represented by Avis constitute the market operation group. ICV development and manufacturing group, market introduction and operation group, together with legislators, insurance companies, infrastructure operators and content providers jointly constitute the ICV industry ecosystem.

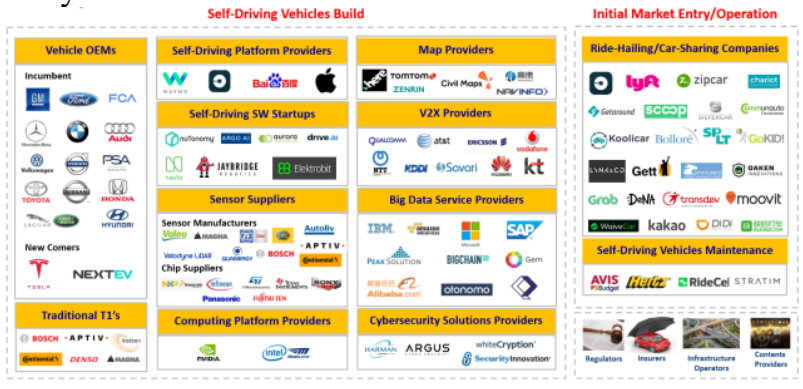

Figure 1. ICV industry ecosystem.

Judging from the composition of the entire ICV industry ecosystem, the automotive industry has shifted from product-centric to service-oriented industry. The first batch of ICVs will also be used for on-demand shared mobility services $[13,14]$ and constantly collect data in the service which will play an increasing role in the future.

\section{Five business models for the ICV industry}

Technology companies and traditional OEMs have poured into the ICV industry ecosystem, and business models have continued to expand from a single business model (car production and sales). Five business models are herein summarized for companies to choose from. They are platform model with technology companies as the mainstay, self-transformation model with OEMs as the mainstay, traditional T1 dependence model, model of alliance between OEMs and technology companies, and
OEM outsourcing model. The business model a company chooses to develop ICVs will profoundly affect how fast it can put ICVs into reality and its future position in the industry.
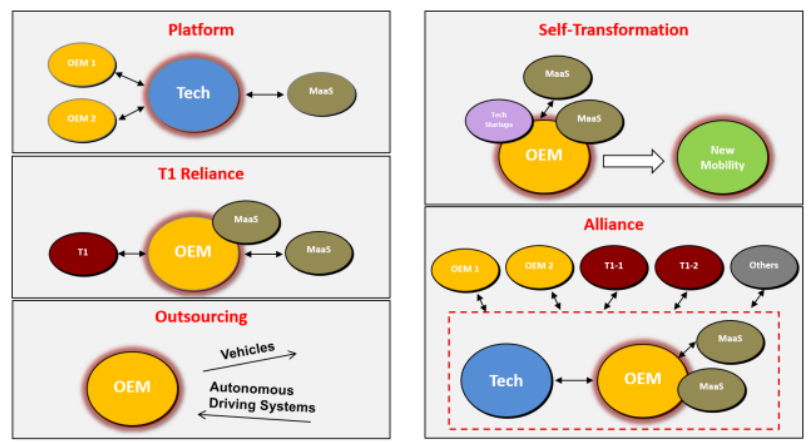

Figure 2. Five Business Models of ICV.

\subsection{Platform model}

The platform model is a business model where technology companies (the mainstay) partner with other enterprises in the ecosystem to provide them with autonomous driving platforms. Representative companies are Waymo, Uber, Baidu and Apple. For example, Waymo partnered with OEMs such as Fiat and Jaguar to provide an autonomous driving platform based on Intel computing hardware. It installed the system on Pacifica hybrid cars and Jaguar I-Pace SUVs to make them become autonomous vehicles, then delivered them to Lyft for operation and Avis for maintenance, and finally recover data therefrom.

Strengths, weaknesses, opportunities and threats (SWOT) matrix is one of the most used tools for strategic planning, especially in the stage of extracting strategies. The use of SWOT analysis is quite common and popular $[15,16]$. Based on the SWOT analysis of the platform model, the internal advantages of this model are: it is conducive to giving full play to the innovative thinking of technology companies and their expertise in software, connectivity technology, and sensors (LiDAR, radar, camera, microphone); at the same time, the autonomous driving systems can rely on OEMs and operation companies to quickly accumulate driving range. Inevitably, the platform model has its internal disadvantages: technology companies do not have selfdeveloped automotive hardware and lack automotive industry experience and market knowledge. From the perspective of the external market environment, there may be opportunities for some OEMs to choose Waymo's hardware and software modules for autonomous driving systems in the early stage of autonomous driving development. In addition, technology companies can develop themselves into transportation companies by virtue of business expansion, OEM acquisition and other methods. Of course, the external threat that the platform model will face is that OEMs will continue to strive to reduce dependencies. Over time, when autonomous driving systems become ordinary commodities, they will lose competitive advantages, because it is impossible for hardware and 
software of autonomous driving systems to achieve a lock-in effect like the Windows operating system. Therefore, the biggest challenge of the platform model is how to maintain a competitive position.

\subsection{Self-transformation model}

The self-transformation model is a business model where OEMs (the mainstay) invest heavily in technology and ride-sharing start-ups, actively develop their own capabilities and enhance their competitiveness. Representative companies are GM, Ford, Audi and Nissan. For example, GM acquired autonomous vehicle startup Cruise and LiDAR sensor startup Strobe. The former has recently received a 2.25 billion USD investment from SoftBank. In addition, in 2016, it invested in the ride-sharing platform Lyft, acquired San Francisco-based car sharing company Sidecar, and strategically cooperated with IBM to introduce IBM's AI technology into its vehicles. Under the effect of capital, GM has independently developed as many autonomous driving systems as possible and launched car-sharing service Maven in 2017 and robo-taxis in 2019. Ford acquired Autonomic, a Silicon Valley-based measurement and architecture solution provider, TransLoc, a software platform supplier, SAIPS, an Israeli machine learning and computer vision technology company, Chariot, a San Francisco-based crowdsourcing service provider, and other autonomous driving and mobility service startups. At the same time, Ford also actively cooperated with artificial intelligence giant IBM, electronics and telecommunications giant Qualcomm, communications giant AT\&T, LiDAR company Velodyne, open source software giant Pivotal, and 3D map startup Civil Maps, and other technology providers on the R\&D of autonomous vehicles, and collaborated with time-sharing rental and Internet car-sharing platforms Zipcar and Lyft, pizza chain Domino's Pizza, and third-party delivery company Postmates to deploy robo-taxi and logistics delivery services.

Through the SWOT analysis, the internal advantage of this model is: OEMs, based on their rich experience in automotive design and manufacturing, feature robust capabilities for autonomous vehicle development, and upon successful $\mathrm{R} \& \mathrm{D}$, are entitled to the intellectual property right and deployment of the autonomous driving systems in the entire value chain, which is conducive to maintaining long-term competitiveness. Inevitably, the self-transformation model also has its internal disadvantage: the initial autonomous vehicle R\&D process of OEMs is slower than that of large technology companies, and the huge development cost burden in the initial stage will also be borne by the OEMs themselves, which will bring great financial pressure. From the perspective of the external market environment, there may be opportunities since this model is attractive to investors with strong financial resources, who feature powerful monetization after the product launch and are more likely to develop into mobility service companies, so the prospects are very bright. The external threats to this model are: if the R\&D fails, the huge investment will not be recovered; if it succeeds, regulatory approvals and market acceptance will be the biggest challenges.

\subsection{Traditional T1 dependence model}

Tradition T1 dependence model is a business model where OEMs and T1 suppliers (the mainstay) cooperate to develop autonomous vehicle. Its representative company is Mercedes-Benz. Mercedes-Benz partnered with Bosch to have two years of exclusive rights to any Bosch-branded products used in its autonomous vehicles. Bosch, based on its cooperation with Tomtom, a Dutchbased company with digital map assets, AutoNavi, a digital map company, Nvidia, a world's leading graphics processor company, Sony, a powerful imaging sensor technology company in Japan, Baidu, an autonomous driving platform company, NavInfo, an IoV and dynamic traffic information service company, etc. assisted Mercedes-Benz in the R\&D of autonomous driving technologies and products. Other than cooperation with T1 Bosch, Daimler also acquired three online car hailing service platforms of RideOut, My Taxi, and e-Hail, and worked with BMW's mobility service department, shared mobility enterprise Uber, peer-topeer car rental platform Getaround and other mobility service providers to launch its own car sharing App Car2go.

Through the SWOT analysis, the internal advantage of this model is long-term partnership between OEMs and $\mathrm{T} 1$ suppliers. Both sides trust each other, which is conducive to integrating T1 suppliers' software, hardware, systems, and test and verification capabilities, giving full play to OEMs' technical expertise in largescale development of safe and efficient vehicles, and allowing OEMs to possess intellectual property rights for a period of time after successful R\&D. In general, this is a less disruptive business model. But since T1 suppliers are under disruption and outstripped by technology companies in the initial development stage, and the entrenched working methods of both sides hinder innovation, OEMs' transformation is slow. From the perspective of the external market environment, the possible opportunity is OEMs' strong monetization of the systems. However, the external threats come, on the one hand, from partners because when the cooperation with T1 is terminated, OEMs will lose control of intellectual property rights, and on the other, from market timing because companies pioneering the successful $R \& D$ of ICVs may have a huge impact on the relevant legislation, making it difficult for other players to launch competitive systems.

\subsection{Alliance Model}

Alliance model is that the OEMs and tech companies form a core alliance and invite other companies to join in to co-develop autonomous vehicles. Its representative company is BMW. BMW and Intel's vision processing company Mobileye form the core of the alliance, inviting OEMs FCA, Tier1 companies Magna, Continental, 
Aptiv, LiDAR company Innoviz, and their respective ecosystems to join the alliance to jointly make deployment of the R\&D of autonomous driving system. On the other hand, it acquired mobility startups Drive Now and Parkmobile, and cooperated with Ridecell, a mobility service software provider, Zirx, an on-demand valet parking company, Scoop, a ride-sharing application company, and zendrive, a mobility big data company.

Using the SWOT matrix, the internal advantage of the alliance model is that alliance members can share high R\&D costs, and each member has different expertise, which can speed up product launch. Inevitably, the model also has internal disadvantages, which is reflected in the weak independent development capabilities of members, which makes it difficult to maintain their own competitive advantages and brand uniqueness. If the $R \& D$ is successful, the extremely complicated relationships between members will make it difficult to effectively manage intellectual property rights. From the perspective of the external market environment, there is an opportunity for the alliance to attract many OEMs to become initial development partners and work together to monetize the system. The external threat is likely to be surpassed by other OEMs with more competitive advantages. If they are only members of the alliance, they may not be able to obtain the host's core technology.

\subsection{Outsourcing model}

The outsourcing model refers to the fact that OEMs rely on tech companies or alliances instead of actively improving their ability to independently develop autonomous driving. The representative company is Fiat Chrysler. Fiat, on the one hand, provides traditional vehicles for Google-owned Waymo, and on the other hand brings additional engineering and technical manpower to the BMW-Intel Alliance, and has never tried to establish leadership alone.

Leveraging the SWOT matrix, the internal advantage of the outsourcing model is that it can enable autonomous vehicles to enter the market faster and obtain early-stage sales returns. Inescapably, the internal disadvantage of this model is the lack of independent expertise and no core intellectual property rights. In terms of the external market environment, the possible opportunity to improve independence ability is to acquire certain partners, but after all, it is still an auto supplier, and may eventually become an automotive hardware supplier. When there are no advantages to be used, it will be at risk of being eliminated.

The five business models include platform model, self-transformation model, reliance on traditional T1 model, alliance model and outsourcing model, each with advantages and disadvantages. Enterprises should choose the appropriate business model based on their existing capabilities and future development positioning, and actively implement it "from one end to the next."

\section{Competition Pattern of ICV Enterprises}

\subsection{The competition pattern is constantly changing}

The global automotive industry and many high-tech companies are actively making deployment of autonomous driving, which gradually constitutes the current competition pattern. Waymo will launch its AVbased ride-hailing service in Arizona, U.S., in the second half of 2020, defeating its closest competitor, GM. GM plans to promote an autonomous Chevrolet Bolt-based ride-hailing service in the second half of next year. The two companies are about to achieve business goals through capacity of independent development and strategic partnerships, and are undoubtedly industry leaders. Fiat Chrysler, Daimler, Audi, Nissan and BMW, with their clear business strategies, have demonstrated near-autonomous-driving capabilities and are leading the industry. Compared with leaders GM and Waymo, Ford, Volvo, Peugeot Citroen, Volkswagen, Tesla and Uber lack the right partnerships, investments or consumer trust, have slow development progress and are fast followers in the field of autonomous driving. While Toyota, Jaguar Land Rover, Honda, Hyundai, Baidu and BAIC are relatively conservative and lack clear and consistent business models, sufficient public road tests, enough partners and investments, and they are followers in the area of autonomous driving. However, Jaguar and Waymo has forged cooperation, and Honda is also discussing cooperation with Waymo. The positions of the two companies may change significantly. However, as a global technology giant, Apple lacks sufficient vision for autonomous driving and future mobility and is a latecomer and a slow follower of autonomous driving. Because of the rapid development of autonomous driving, the competition pattern of autonomous driving has not yet been finalized and is changing every day.

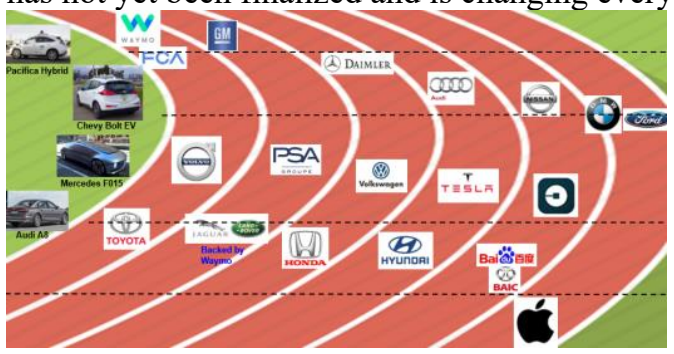

Figure 3. Competition Pattern of ICV Enterprises

\subsection{Five factors to maintain competitive advantages}

In a constantly changing competition pattern, how should companies develop or maintain competitive advantages? In general, companies can develop or maintain competitive advantages from five perspectives: technology, market timing, customer experience, brand and data. In the short term, the selection of business model will directly impact the technological competitiveness of the enterprise. The fully independent development of dedicated autonomous driving technology is the most critical differentiating factor. If the in-house developed autonomous driving system is more advanced than the systems of other companies, it 
will naturally own strong competitive advantages. If there is no in-house development at all, but a direct purchase of a universal autonomous driving system without intellectual property rights, it will not bring any technical advantages. No matter who gets the market first, it will be in a good position to invest more and continue to innovate faster than its competitors. In the long run, autonomous driving systems will eventually be commoditized, and then it will be difficult to enhance their competitive advantages via technologies.

When the key technical conditions are equal, a solution that considers human basic attributes and needs and provides a personalized riding experience will become the key to differentiation. The value of one vehicle relative to another will be differentiated based on the convenience, comfort, and customized services provided to passengers, and companies that provide consumers with a high level of service and are able to combine supply and demand are likely to create maximum value and profit.

Brand building and market segmentation remain important. Uber black, Uber pool, and UberX services are also available in the mobility service field for different market segments. As a result, premium brands have specific markets in terms of mobility as a service (Maas) and private autonomous vehicles for the rich. In the future, firms will face different markets, different demands and completely different customer groups. Players that have capabilities to provide things needed in different market segments will have competitive advantages. In the intelligent vehicle segment, data will evolve from "best to own" into a key differentiator for survival of enterprises. In the future, data will become an important means of production, and enterprises with core data will feature competitive advantages.

\section{Conclusion}

Although ICVs have become an irresistible "inevitability", there are still an array of social and technical obstacles on the way forward. This unprecedented challenge makes the traditional vertical supply chain of the automotive industry have to give way to the horizontal cooperation model of softwarehardware collaborative development. At the same time, manufacturing costs and complexity have also risen, forcing business models to shift from traditional "buying and selling" to "cooperation." In this new form, each player will bring technical strength, intellectual property rights, design expertise, manufacturing capabilities, research inputs, equity participation and other strategic contributions, take corresponding risks and get due returns. There are different technology paths and business models for bringing ICVs to the market. But in order to choose the most suitable path, enterprises must recognize their current position, technical and financial potential, future development goals, and the national conditions of the country in which they are located.

In terms of business model selection, if enterprises want to get a fat chunk from the ICV market and maintain long-term competitive advantages, they should choose a relatively independent business model and build internal capabilities as soon as possible to adapt to the new mobility environment, and as early as possible To enter the L4 market. If an enterprise has neither internal resources nor external assistance to build its own capabilities, the outsourcing model can bring its ICVs to the market earlier and reap the early-stage sales results. However, it should be noted that improving the competitiveness of the company must always be the first priority. If the status of the company does not improve during this process, it may eventually become a foundry for others. In the long term, enterprises with clear and firm directions as well as relatively simple partnerships will make significant progress. Without a clear direction, playing pendulum, or with complex business cooperation models, participating in too many alliances can only slow down the pace of business.

\section{Acknowledge}

This research is supported by the National Natural Science Foundation of China (U1764265).

\section{References}

1. Zhou, J, China Mech. Eng, 26(17), 2273(2015)

2. Zhao F. Auto Manu. Eng, 12, 16(2016)

3. Kuang X, Zhao F, Hao H, et al, Sustainability, 11(12): 3273(2019)

4. Tan H, Zhao F, Hao H, et al, Traffic Inj.Prev, 1(2020)

5. Tan H, Zhao F, Hao H, et al. Int. J. Environ. Res. Public Health, 17(3): 917(2020)

6. SAE J3016(TM). SAE Int,18-19 (2018)

7. Liu Z, Tan H, Hao H, et al. MATEC Web of Conferences. EDP Sciences, 296: 01002(2019)

8. Zhao F, Liu Z. Forum on Science and Technology in China, 1: 58-68(2016).

9. Toglaw S, Aloqaily M, Alkheir A A. 2018 Fifth International Conference on Internet of Things: Systems, Management and Security. IEEE, 303308(2018)

10. Liu Z. China Machine Press: Beijing, China(2017)

11. Yun J H J, Won D K, Jeong E S, et al. Technological Forecasting and Social Change, 103: 142-155(2016)

12. Kuang X, Zhao F, Hao H, et al. Asia Pac. Bus. Rev., 24(1): 1-21(2018).

13. Renneby, Victor, and Johannes Sommer. "The Transition to Autonomous - Impact \& Challenges in the Race toward Self-Driving Cars." (2018).

14. Bernhart W. ATZelektronik worldwide, 11, 36(2016)

15. Sabbaghi A, Vaidyanathan G. Information systems education journal, 2(23): 3-19(2004)

16. Ebonzo A D M, Liu X. Qual. Quant., 47(5): 26712685(2013) 